Research Paper

\title{
Massive Production of All-iemale Diploids and Triploids in the Crucian Carp
}

\author{
KaiKun Luo*, Jun Xiao*, ShaoJun Liu ${ }^{\bowtie}$, Jing Wang, WeiGuo He, Jie Hu, QinBo Qin, Chun Zhang, Min Tao \\ and Yun Liu
}

Key Laboratory of Protein Chemistry and Developmental Biology of State Education Ministry of China, College of Life Sciences, Hunan Normal University, ChangSha, 410081, China.

* These authors contributed equally to this work.

Corresponding author: Tel: +86 731 88872552; fax: +86 731 88873074. (E-mail: 1sj@hunnu.edu.cn).

( ) Ivyspring International Publisher. This is an open-access article distributed under the terms of the Creative Commons License (http://creativecommons.org/ licenses/by-nc-nd/3.0/). Reproduction is permitted for personal, noncommercial use, provided that the article is in whole, unmodified, and properly cited.

Received: 2011.01.01; Accepted: 2011.04.01; Published: 2011.04.16

\begin{abstract}
In many species of aquaculture importance, all-female and sterile populations possess superior productivity due to faster growth and a relatively homogenous size of individuals. However, the production of all-female and sterile fish in a large scale for aquaculture is a challenge in practice, because treatments necessary for gynogenesis induction usually cause massive embryonic and larval mortality, and the number of induced gynogens is too small for their direct use in aquaculture. Here we report the massive production of all-female triploid crucian carp by combining artificial gynogenesis, sex reversal and diploid-tetraploid hybridization. Previously, we have obtained an allotetraploid carp population $(4 n=200)$ by hybridization between red crucian carp (Carassius auratus red var; + ) and common carp (Cyprinus carpio; $\delta$ ). We induced all-female diploid gynogens of the Japanese crucian carp (Carassius cuvieri; $2 n=$ 100). We also generated male diploid gynogens of the same species treated gynogenetic fry with 17-a-methyltestosterone, leading to the production of sex-revered gynogenetic males. Finally, these males were used to cross with the female diploid Japanese crucian carp gynogens and the allotetraploid females, resulting in the production of fertile all-female diploid Japanese crucian carp $(2 n=100)$ and sterile all-female triploid hybrids $(3 n=150)$, respectively. Therefore, diploid crucian carp gynogenetic females and sex-reversed male together with an allotetraploid line provide an opportunity to produce all-female triploid populations in a large scale to meet demands in aquaculture industry.
\end{abstract}

Key words: gynogenesis, sex reversal, all-female diploid, all-female triploid, Stem cell, fish breeding.

\section{Introduction}

In many species of cultured finfish, females exhibit higher growth rates than males and attain larger sizes, and males mature and slow down growth before reaching a marketable size. Differences in growth rate and timing of sex maturation result in a mixed population of individuals showing considerable variations in sizes and consequently an overall reduction in production. Therefore, there is an increasing inter- est in generating all-female brood stocks for cost-efficient aquaculture [1]. Gynogenesis is an important breeding method in fish and has been widely applied to improve the genetic characteristics of various fish species [2, 3]. Gynogenesis is all-female development without genetic contribution of sperm. Gynogenesis occurs frequently in natural populations of various lower vertebrates [4-7]. Gynogenesis can also 
be artificially induced by inseminating eggs with genetically inactivated sperm, followed by the suppression of second polar body extrusion and first cleavage, leading to meiotic and mitotic diploid gynogenesis, respectively. The resultant diploid gynogenetic fish have the ability to grow to the adulthood and mature for reproduction. Diploid gynogens show either unisexuality or bisexuality depending on the genetic determination system. In fish, there is a diverse array of sex determination systems including the $X X / X Y$ female homogamy/male heterogamy and the $\mathrm{ZZ} / \mathrm{ZW}$ male homogamy/female heterogamy $[8,9,10]$. In female-homogametic species such as the crucian carp, gynogenesis - either natural or artificial - usually produces all-female populations [11, 12, 13]. Therefore, artificial gynogenesis is a useful tool for all-female broodstock production in otherwise bisexually reproducing fish species.

Natural gynogenesis involves the participation of both normal sperm and eggs, and embryogenesis in the absence of genetic contribution of sperm occurs normally, leading to an efficiency way for progeny production comparable to bisexual reproduction. Artificial gynogenesis, however, faces one major challenge for direct use in brood production. To induced artificial gynogenesis, eggs are subject to pulse treatments at a critical stage to prevent second polar body extrusion in meiosis II or first cleavage. These treatments are usually physical shocks, namely thermal (cold or heat) shocks [14-18] and pressure shocks [19, 20]. These shocks cause damages to eggs and thus affect embryonic and larval development, leading to massive lethality, limiting the usefulness of artificial gynogenesis for large-scale production in aquaculture.

Another approach that has widely been used for direct induction of all-female fish is sex reversal by the hormonal treatment $[1,21,22]$. This approach has a similar problem as mentioned for artificial gynogenesis, as there are several factors that may limit its direct use for large-scale production. Hormonal treatment does not guarantee a $100 \%$ efficiency of sex reversal for female production, and fry can be used only once, making this approach costly and time consuming [23]. In addition, there is a concern from the market on any side-effect of a hormone-treated food product.

The combination of artificial gynogenesis and sex-reversal with crossing between normal female fish and genetically female but sex-reversed male fish has attracted considerable attention for the reproducible production of all-female fish to meet the requirement for massive production of all-female fish in aquaculture [24-27]. However, there has been no report on the successful establishment of all-female crucian carp production by combining gynogenesis and sex re- versal.

In our previous study, a population of fertile AT was obtained by crossing red crucian carp (Carassius auratus red var, +) $\times$ common carp (Cyprinus carpio, ô) [28]. So far, this population has propagated 16 generations $\left(F_{3}-F_{18}\right)$ [29]. Triploid crucian carp we call 3nCC $(3 n=150)$ were then produced by mating AT $(\widehat{\jmath})$ with JCC (Carassius cuvieri, o), which combined sterility and obvious hybrid vigor or heterosis in aquaculture. Now more than 400 million 3nCC are annually produced for aquaculture in China [28,30]. We have also taken one additional step to improve the 3nCC productivity by generating superior Japanese crucian carp individuals as the parental strain of hybridization. To this end, we made use of induced gynogenesis and obtained diploid JCC gynogens that are superior in disease resistance, hypoxia tolerability and adaptability ${ }^{[31]}$. However, the number of superior JCC gynogens was small and unable to satisfy the request in aquaculture.

In this study, a large population of $2 \mathrm{nFCC}$ was obtained by crossing the RGJCC ( ${ }^{1}$ ) with the GJCC (). Furthermore, a new all-female triploid crucian carp named 3nFCC was generated by hybridizing the RGJCC ( $(0)$ with AT (). There have been few reports of all-female triploid fish [32]. Previously, to form triploid fish, it was necessary to artificially suppress the second meiotic division in the ovum after fertilization, which could not make sure to producing $100 \%$ triploid progeny. In trout, Daniel Chourrout induced a tetraploid line by heat shock and used it to produce triploids for production in France [14], but as mentioned above, tetraploidy induced by heat shock was always associated with high mortality rates, limiting the large-scale production of triploids. By contrast, the present study produced the all-female triploid hybrids by crossing RGJCC ( $\hat{0})$ with AT (ㅇ). This method allows for the production of all-female triploids on a large scale. It is the first report for the establishment of an all-female triploid by the combination of gynogenesis, sex reversal and interspecific mating.

The formation of all-female diploid and triploid crucian carp provides the excellent platform to investigate the sex differentiation of the primordial germ cells (PGCs) [33]. In this aspect, we clearly know the PGCs of all-female diploid and triploid crucian carp will finally develop into oogonia and mature ova [34]. So we can observe how the PGCs of the female diploid and triploid undergo the sex differentiation.

On the other hand, the formation of all-female diploid and triploid crucian carp also provides the excellent platform to perform the nuclear transfer. We can transfer the nucleus of the blastula from the 
all-diploid or triploid crucian carp into the fertilized egg whose nucleus is moved out. So, in theory, the "nuclear-transferred" egg will develop into the female diploid or triploid individual $[35,36,37]$. If we get all female diploid or triploid individuals, we can conclude that the "nuclear-transferred" experiments are successful.

After the formation of all-female diploid and triploid crucian carp, we can also use them to study the signal path of some sex correlation genes, especially the important sex determining genes of female in crucian carp. First, establish all-female PGC lines in vitro $[38,39,40]$. Then, we can knock out the target gene in the cell line and transfer the nucleus of the all-female PGC to the zygote whose nucleus is moved out and observe the affects of the target gene [41,42]. If the target gene plays an important role in the female sex determination, the changes in the offspring of the "nuclear-transferred" eggs may be easily observed and analyzed since there is no interference of the male sex determining genes in the all-female crucian carp.

Thus, the formation of all-female diploid and triploid crucian carp provides good experimental material for fish stem cell and nuclear transfer researches.

\section{Material and methods}

All samples, including JCC, GJCC, RGJCC, 2nFCC, 3nFCC, 3nCC, AT, and Blunt snout bream (Megalobrama amblycephala, abbreviated as BSB) were collected from the Engineering Center of Polyploid Fish Breeding of the Education Ministry, China, located at Hunan Normal University. Animal handling and experimentation followed the Interdisciplinary Principles and Guidelines for the Use of Animals in Research, Testing, and Education of the New York Academy of Sciences, USA.

\section{Production of GJCC and RGJCC}

Spawning female JCC and male BSB were used for artificial propagation [31]. For artificial gynogenesis, the semen of BSB was stripped, diluted with Hank's solution (1:4), poured into a glass dish on ice and exposed to UV irradiation at $2 \mathrm{~mW} / \mathrm{cm}^{2}$. The spermatozoa suspension was continuously stirred by rocking during the irradiation. Total UV dosages administered were $3600 \mathrm{~mJ} / \mathrm{cm}^{2}$, which corresponded to an irradiation duration of $30 \mathrm{~min}$. Ova stripped from JCC were then inseminated with the irradiated sperms. Two minutes later, the inseminated eggs were subjected to a cold shock at $0-4^{\circ} \mathrm{C}$ for $40 \mathrm{~min}$ to prevent second polar body extrusion. The eggs were then incubated at $18-20^{\circ} \mathrm{C}$ in fresh water. Approximately 1000 fry at day 2 post hatching were treated with 17-a-methyltestosterone (MT) through oral administration of diet at a dose of $100 \mathrm{ug} / \mathrm{g}$ for 60 days. Control fry were fed with the normal diet without MT.

\section{Gonadal histology}

After growth for 4 months, 10 MT-treated GJCC fish were randomly sampled to detect sex reversal by histological sectioning. Briefly, the gonads was fixed in Bouin's solution, embedded in paraffin, sectioned and stained with hematoxylin and eosin. Gonadal structures were observed and photographed with a Pixera Pro 600ES. The gonad was staged according to the Liu's standard series of cyprinid fish [2]. In the second spawning season, 30 RGJCC were striped to monitor for semen production. The semen was collected with a clean sucker and prefixed in $2.5 \%$ glutaraldehyde solution for monitoring morphology. The samples were centrifuged at $2000 \mathrm{rpm} / \mathrm{min}$ for $1 \mathrm{~min}$ on an Eppendorf centrifuge 5804R, fixed in $4 \%$ glutaraldehyde solution overnight, and refixed in $1 \%$ osmic acid solution for 2 hours. The samples were dehydrated in ethanol, dropped onto slides, desiccated, and subjected to atomized gilding before observation on an X-650 (HITACHI) SEM scanning electron microscope.

Observation of gonadal structure of $2 \mathrm{nFCC}$ and $3 \mathrm{nFCC}$ was similarly performed, except for that 20 2nFCC and $203 \mathrm{nFCC}$ fish were used at the age of 7 months.

\section{Production of $2 \mathrm{nFCC}$ and $3 \mathrm{nFCC}$}

At spawning seasons, 30 RGJCC males, 30 GJCC males and 30 AT females were used for the production of 2 nFCC and $3 \mathrm{nFCC}$. Fifteen RGJCC males were used to mate with the 30 GJCC females and the other 15 RGJCC males with the 30 AT females. JCC and 3nCC were also produced at the same time as control groups. To check the fertility rate, 10 duplicates of about 600 fertilized eggs in each cross were placed in a glass dish. The eggs were reared in well water at $20^{\circ} \mathrm{C}$. The average fertility rate (number of gastrula/total number of eggs $\times 100 \%$ ) and hatching rate (number of hatchlings / total number of eggs $\times 100 \%$ ) were counted. For both fertility rate and hatching rate, a t-test was used to analyze the covariance of the data between 2 nFCC and JCC, 3nFCC and 3nCC. The hatched fry were grown in ponds.

\section{Examination of ploidy levels}

To determine ploidy levels, chromosome preparations were obtained from peripheral blood cell cultures of 7-month-old fish. Briefly, $0.2 \mathrm{ml}$ of blood sample was collected by using a syringe soaked with $0.1 \%$ sodium heparin, cultured in nutrient solution at 
$25.5^{\circ} \mathrm{C}$ and $5 \% \mathrm{CO}_{2}$ for $68-72$ hours, and colchicine was added $3.5 \mathrm{~h}$ before harvest. Cells were harvested by centrifugation, followed by hypotonic treatment with $0.075 \mathrm{~m} \mathrm{KCl}$ at $26^{\circ} \mathrm{C}$ for $25-30 \mathrm{~min}$, followed by fixation in 3:1 methanol-acetic acid with three changes. Cells were dropped on cold slides, air-dried and stained for $30 \mathrm{~min}$ in $4 \%$ Giemsa solution. Chromosomes were examined under a microscope. For each type of fish, 100 metaphases (10 metaphase each sample) of chromosomes were analyzed.

\section{Results}

\section{Chromosomes of GJCC, RGJCC, 2 FFCC, and 3nFCC}

Chromosomes were counted in 10 metaphases in each sample of GJCC, RGJCC, $2 \mathrm{nFCC}$, and $3 \mathrm{nFCC}$ (Table 1). Chromosome number ranged from 95 to 100 (accounting to 97 98\%) in GJCC (Fig. 1a), RGJCC (Fig. $1 \mathrm{~b})$ and 2 nFCC (Fig. 1c), indicating that they were diploid $(2 n=100)$. As expected, $3 n F C C$ was triploid $(3 n$ $=150)$, as evidenced by a predominate proportion (94\%) of metaphases of 145 to 150 chromosomes (Fig. 1d).

Table I Distribution of chromosome numbers in mitotic metaphase

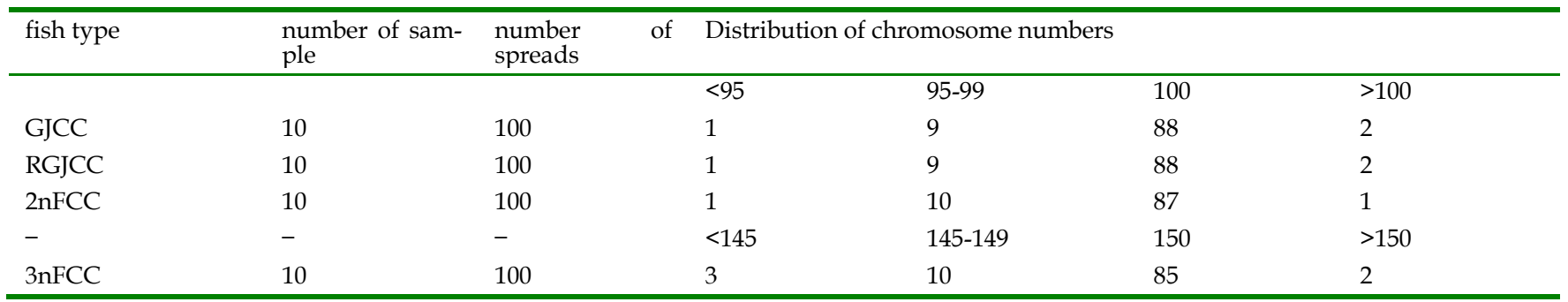
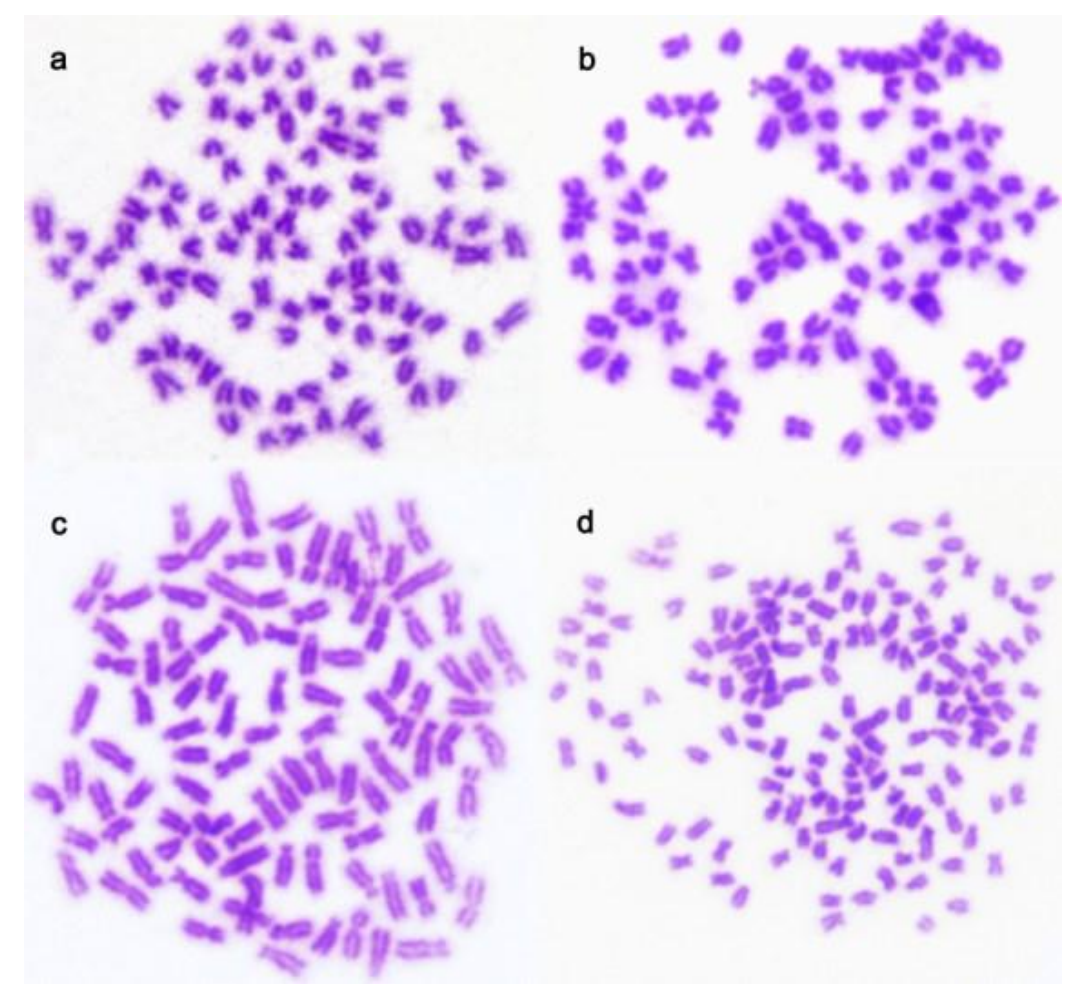

Fig. I Metaphase. (a) Gynogenetic Japanese crucian carp (GJCC) showing $2 n=100$. (b) Sex-reversed gynogenetic Japanese crucian carp (RGJCC) showing $2 n=100$, (c) All-female diploid Japanese crucian carp $(2 n F C C)$ showing $2 n=100$. (d) All-female triploid crucian carp (3nFCC) showing $3 n=150$. 


\section{Efficiency of sex reversal}

After MT treatment, GJCC developed two types of gonadal structure (Fig. $2 \mathrm{a}, \mathrm{b}$ ). Of the 10 fish observed, 9 exhibited complete sex reversal to functional males, as evidenced by a clear testicular structure on sections, and 1 was incompletely sex reversed, as evidenced by an ovotesticular structure. This observation indicates a high 90\% efficiency of MT-induced female-to-male sex reversal in GJCC. Concurrently, white semen could be stripped out from $93.3 \%$ of the MT-treated GJCC fish. Sperms from these sex-reversed GJCC, namely RGJCC males, were normal in morphology, as they had a well-developed head and tail under the scanning electron microscope (Fig. 2 c, d).

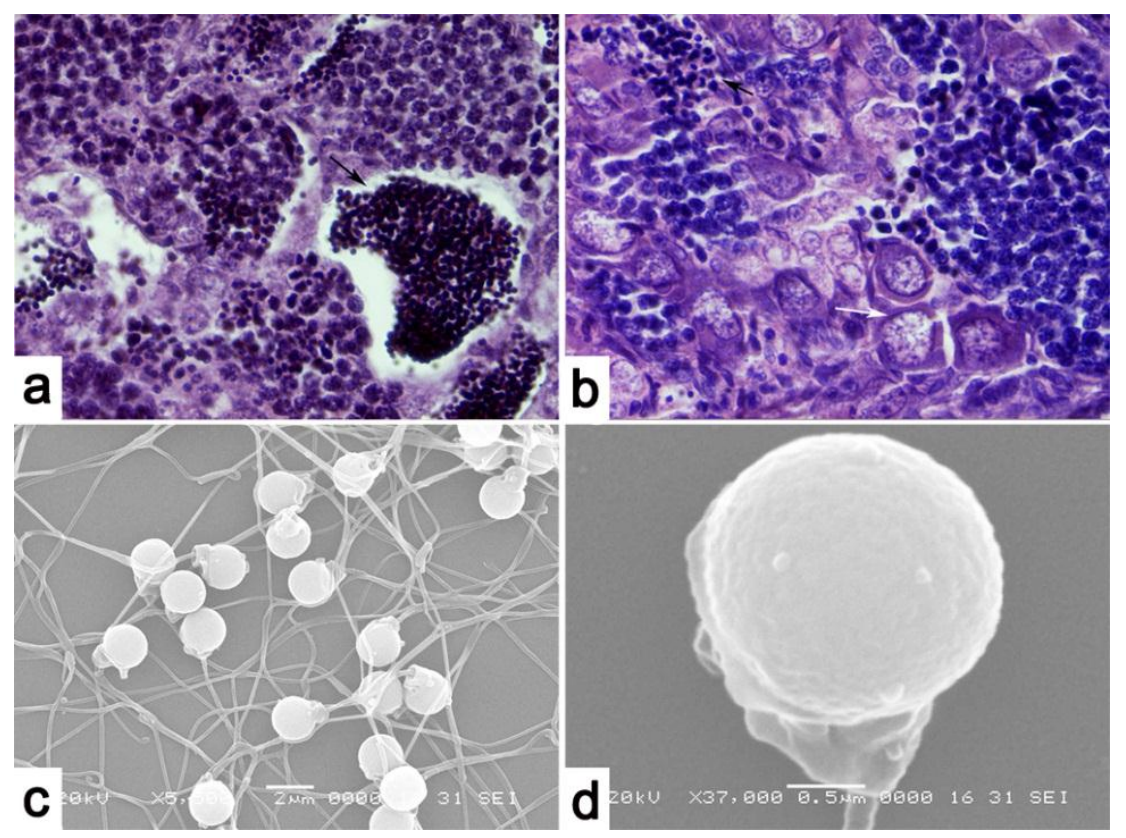

Fig. 2 Structures of gonads and sperm of Sex-reversed gynogenetic Japanese crucian carp. (a) Testis structure of RGJCC, the black arrow shows abundant mature sperm in seminiferous tubules. (b) Testis and ovary structure of RGJCC, the black arrow shows sperm, the white arrow shows the primary oocyte. (c) The structure of sperm of RGJCC under the scanning electron microscope, bar=2um. (d) Structure of the sperm of RGJCC under the scanning electron microscope, bar=0.5um.

\section{Fertility rate and hatch rate}

The average fertility rate and hatching rate of 2nFCC were $90.6 \%$ and $83.9 \%$, respectively (Table 2 ). There were no differences between $2 \mathrm{nFCC}$ and the control JCC ( $p>0.05)$. 3nFCC showed the same characteristics. These results demonstrate that RGJCC can produce functional sperm.

Table 2 Fertility and hatching rates

\begin{tabular}{lllll}
\hline & JCC & 2nFCC & 3nCC & 3nFCC \\
\hline $\begin{array}{l}\text { Fertility } \\
\text { rate }(\%)\end{array}$ & $91.2 \pm$ & $90.6 \pm 1.04$ & $86.5 \pm 1.41$ & $85.4 \pm 1.33$ \\
$\begin{array}{l}\text { Hatch rate } \\
(\%)\end{array}$ & $85.3 \pm$ & $83.9 \pm 1.02$ & $81.7 \pm 1.26$ & $80.8 \pm 1.55$ \\
\hline
\end{tabular}

\section{Gonadal development of $2 \mathrm{nFCC}$ and $3 \mathrm{nFCC}$}

At the age of seven months, the gonadal structures of 20 randomly selected 2 nFCC and 203 nFCC were observed by histological sections. As shown in Fig. $3 a$, the ovaries of $2 \mathrm{nFCC}$ were in stage IV and the oocytes were rich in yolk materials. In addition, during the reproduction season, green ova were able to be stripped from $2 \mathrm{nFCC}$. These results indicated that the 2 nFCC were fertile. Interestingly, only one type of gonadal structure was observed in 20 samples of 7-month-old 3nFCC. All the gonads were ovary-like gonads containing many oogonium-like cells and very few cells in II-stage (Fig. $3 b$ ), suggesting that 3nFCC are sterile. 


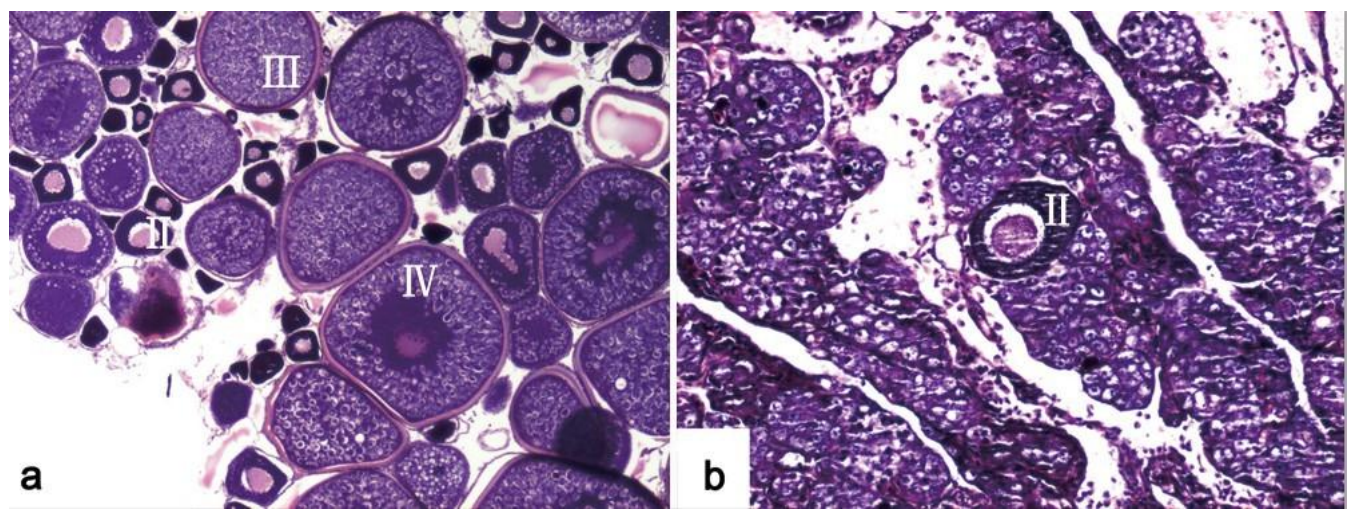

Fig. 3 Ovarian development. (a) All-female diploid Japanese crucian carp $(2 \mathrm{nFCC})$. The histological section shows the oocytes at stages form II- IV. The labels depict primary oocytes at different stages. (b) All-female triploid crucian carp ( $3 \mathrm{nFCC})$. The histological section shows the presence of very few oocytes of early stage. The label depicts a primary oocyte at stage II.

\section{Discussions}

\section{The formation of a genetically inbred line of $2 n F C C$}

Gynogenesis is an important method in fish genetic breeding. It is effective for obtaining genetically inbred fish lines. Most economic traits of fish are controlled by multiple genes models. More than ten generations are usually needed to gain an inbred line by conventional brother-sister crossing. A similar level of inbreeding can be achieved by a single generation of gynogenesis and sex reversal [9, 43]. Inbreeding may lead to the homozygosity of many loci and thus the elimination of many harmful recessive genes. Moreover, there are allogynogenetic effects that might lead to differences between the mother and progeny in appearance, growth rate and other characteristics [44-47]. For example, all-female GJCC directly from gynogenesis already show certain improved traits [31]. However, only a few fry were obtained due to the low survival rate. To increase the survival rate and enlarge the gynogenesis population, the method of sex reverse was employed in this study. In homogametic female fish, all-female progenies could be obtained on a large scale by mating normal females (XX) or gynogenetic individuals $(X X)$ with sex reversal males $(X X)$. The production of functional sex reversal males played an important role in the formation of all-female fish.

The dietary treatment and immersion were the most acceptable methods for administering steroids and 17-a-methyltestosterone was the most preferred hormone for induction of masculinization [23, 48]. The dose of sex hormones and the timing and duration of the administration are important factors in achieving sex-reversed fish $[1,48]$. In this study, the GJCC fry were treated with MT two days after hatching. Histological sections, semen structures under the scanning electron microscope, and crossing experiments proved that the RGJCC were functional males. Intersexual individuals were observed in RGJCC, which is consistent with the regular observation of incomplete sex transformations during steroid treatments in fish ${ }^{[8]}$. The high reversal rate of $93.3 \%$ indicated that this method was effective in inducing sex reversal of Japanese crucian carp.

By the combination of gynogenesis and sex reversal, a large number of fertile $2 \mathrm{nFCC}$ can be obtained by crossing the GJCC or 2 nFCC with RGJCC. RGJCC can also be obtained simply by feeding 2 nFCC fry with MT-containing diet. The generation of RGJCC enables the cycled production of $2 \mathrm{nFCC}$ in a large scale (Fig. 4). The formation of a consecutive line of diploid 2nFCC produced a population that will be useful for genetic improvement of JCC. 


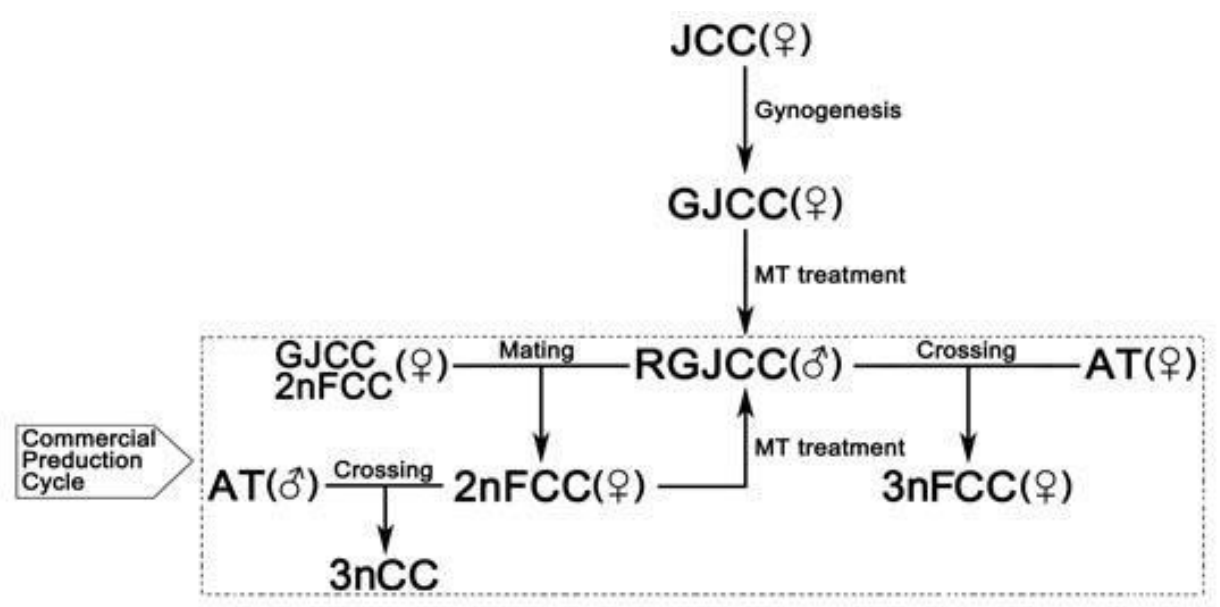

Fig. 4 The origins of each kind of crucian carp

\section{Formation of a new type of all-female triploid population}

With the diploid gametes stably generated by AT, several kinds of sterile triploid crucian carp (3nCC) were produced by mating males of allotetraploids with females of diploid crucian carp. These triploids had some enhanced performances such as sterility, fast growth rate, good flesh quality, and strong stress resistance, and they were widely cultured in China [30]. Our previous studies found that there were three types of gonadal structure in 3nCC, ovary-like gonads, testis-like gonads, and fat-like gonads, and the 3nCC with ovary-like gonad grew faster than those with testis-like gonads [49]. Thus, it is necessary to produce the all females triploid so as to increase the yield. In this study, 3 nFCC were obtained on a large scale by crossing RJGCC with female AT (Fig. 4). The gonadal histological sections of all 20 seven-month-old 3nFCC were ovary-like gonads. As shown in Fig. $3 \mathrm{~b}$ the ovary-like gonads of 3nFCC contained many oogonium-like cells and very few cells in II-stage, while ovaries of the fertile 2nFCC were full of stage IV oocytes (Fig.3a), which is similar to our previously reported sterile $3 \mathrm{nCC}$. This indicated that $3 \mathrm{nFCC}$ were sterile. There have been few reports on the formation of all-female triploids [32]. However, in these studies, physical or chemical treatments were used to suppress the elimination of the second polar body. Incompletely polyploidization, complicated methodology and high costs limited the large-scale production of all-female triploid with these methods. Our study provided a new way for producing all-female triploids.

\section{Biology significance of the formation of the two kinds of all-female crucian carp}

All-female fish, as a monosex population, provide good material for research on the reproductive physiology of fish. The formation of $2 \mathrm{nFCC}$ lays the foundation for the breeding of improved JCC. RGJCC is a good example for the study of early gonad development of crucian carp and the production of 3nFCC opens a new method for all-female production.

In terms of food safety, the production of all-female fish by a combination of gynogenesis and sex reversal is preferred as there is no need to treat any reared fish with a hormone, especially as hormonally treated fish are not intended for human consumption. Ecologically, the number of reared fish will not rise as $2 \mathrm{nFCC}$ cannot breed by self-mating, and $3 \mathrm{nFCC}$ cannot propagate due to its sterility and thus will not abolish genetic resource.

\section{Abbreviations}

JCC: Japanese crucian carp; GJCC: Gynogenetic Japanese crucian carp; RGJCC: Sex-reversed gynogenetic Japanese crucian carp; AT: Allotetraploid hybrid; 2nFCC: All-female diploid Japanese crucian carp; 3nFCC: All-female triploid crucian carp; 3nCC: Triploid crucian carp; BSB: Blunt snout bream.

\section{Conflict of Interests}

The authors have declared that no conflict of interest exists.

\section{References}

1. Piferrer F. Endocrine sex control strategies for the feminization of teleost fish. Aquaculture. 2001; 197: 229- 281. 
2. Liu Y. Propagation Physiology of Main Cultivated Fish in China. Beijing, China: Agricultural Publishing House; 1993.

3. Arai K. Genetic improvement of aquaculture finfish species by chromosome manipulation techniques in Japan. Aquaculture. 2001; 197: 205-228.

4. Cherfas NB. Natural triploidy in the females of the unisexual variety of the goldfish Carassius auratus gibelio (Bloch). Sov Genet. 1966; 13: 557-563.

5. Cherfas NB, Gomelsky BI, Emelyanova OV, et al. Induced diploid gynogenesis and polyploidy in crucian carp, Carassius auratus gibelio (Bloch) X common carp, Cyprinus carpio L hybrids. Aquacult Fish Manage. 1994; 25: 943-954.

6. Dong S, Taniguchi N, Tsuji S. Identification of clones of ginbuna Carassius langsdorfi by DNA fingerprinting and isozyme pattern. Nippon Suisan Gakkaishi. 1996; 62: 747-753.

7. Zhang Q, Arai K. Flow cytometric DNA contents of somatic cells and spermatozoa in the progeny of natural tetraploid loach. Fish Sci. 1996; 62:870-877.

8. Devlin RH, Yoshitaka N. Sex determination and sex differentiation in fish: an overview of genetic, physiological, and environmental influences. Aquaculture. 2002; 208: 191-364.

9. Streisinger GC, Walker N, Dower DK, et al. Production of clones of homozygous diploid zebra fish (Brachydanio rerio). Nature. 1981; 291:293-296.

10. Castelli M. Study on sex determination in the common barbel (Barbus barbus L.) (Pisces, Cyprinidae) using gynogenesis. In: Beaumont AR, ed. Genetics and Evolution of Aquatic Organisms. London, UK: Chapman and Hall, 1994:509-519.

11. Stanley JG. Female homogamety in grass carp (Ctenopharyngodon idella) determined by gynogenesis. J Fish Res Bd Can. 1976; 33:1372-1374

12. Nagy A, Rajki $\mathrm{K}$, Horváth $\mathrm{L}$, et al. Investigation on carp, $C y$ prinus carpio L. gynogenesis. J Fish Biol. 1978; 13: 215-224.

13. Pongthana N, Penman DJ, Karnasuta J, et al. Induced gynogenesis in the silver barb (Puntius gonionotus Bleeker) and evidence for female homogamety. Aquaculture. 1995; 135: 267-276.

14. Chourrout D. Tetraploidy induced by heat shock in the rainbow trout. Reprod Nutr Dev. 1982; 22:569-574

15. Bidwell CA, Chrisman CL, Libey GS. Polyploidy induced by heat shock in channel catfish. Aquaculture, 1985; 51:25-32.

16. Hong YH. Tetraploidy induced by heat shock in bighead carp, Aristichthys nobilis. Acta Zoologica Sinica.1990, 36(1):70-76.

17. Don J, Avtalion PR. Comparative study on the induction of triploidy in tilapias, using cold- and heat-shock techniques. J Fish Bio. 1988; 32: 665-672.

18. Hussain MG, Chatterji A, McAndrew BJ, et al. Triploidy induction in Nile tilapia, Oreochromis niloticus L. using pressure, heat and cold shocks. TAG. 1991; 81: 6-12.

19. Chourrout D. Pressure-induced retention of second polar body and suppression of first cleavage in rainbow trout: Production of all-triploids, all-tetraploids, and heterozygous and homozygous diploid gynogenetics. Aquaculture. 1984;:111-126

20. Volckaert FAM, Galbusera PHA, Hellemansa BAS, et al. Gynogenesis in the African catfish (Clarias gariepinus). I. Induction of meiogynogenesis with thermal and pressure shocks. Aquaculture. 1994; 128: 221-223.

21. Shelton WL, Hopkins KD and Jensen GL. Use of hormones to produce monosex Tilapia for aquaculture. In: Smitherman RO, Shelton WL and Grover JH, eds. Culture of exotic fishes symposium proceedings Fish Culture Section. Auburn, Alabama, USA: American Fisheries Society. 1978: 10-33.

22. Komen J, Lodder PAJ, Huskens F, et al. Effects of oral administration of 17 alpha-methyltestosterone and 17 beta-estradiol on gonadal development in common carp, Cyprinus carpio L. Aquaculture.1989; 78:349-363.

23. Pandian TJ, Sheela SG. Hormonal induction of sex reversal in fish. Aquaculture. 1995; 138: 1-22.
24. Nagy A, Bercsényi M, Csányi V. Sex reversal in carp (Cyprinus carpio) by oral administration of methyltestosterone. Can J Fish Aquat Sci. 1981; 38:725-728.

25. Boney SE, Shelton WL, Yang SL, et al. Sex reversal and breeding of grass carp. Trans Am Fish Soc. 1984; 113:348-353.

26. Shelton WL. Control of sex for cyprinids in aquaculture. In: Billard R, Marcel J, eds. Aquaculture of Cyprinids. Paris, France: INRA. 1986:179-194.

27. Wu C, Chen R, Ye Y, et al. Production of all-female carp and its applications in fish cultivation. Aquaculture. $1990 ; 85: 327$.

28. Liu S J, Liu Y, Zhou GJ, et al. The formation of tetraploid stocks of red crucian carp $\times$ common carp hybrids as an effect of interspecific hybridization. Aquaculture. 2001; 192: 171-186.

29. Chen S, Wang J, Liu SJ, et al. Biological characteristics of an improved triploid crucian carp. Sci China Ser C-Life Sci. 2009; 52(8):733-738.

30. Shen JM, Liu SJ, Sun YD, et al. A new type of triploid crucian carp-red crucian carp $(+) \times$ allotetraploid( $\left({ }^{\wedge}\right)$. Prog Nat Sci. 2006; 16: $1348-1352$

31. Sun YD, Zhang C, Liu SJ, et al. Induction of Gynogenesis in Japanese Crucian Carp (Carassius cuvieri). Acta Gene Sinica. 2006; 33(5):405-412.

32. Rothbard S, Shelton WL, Rubinshtein I, et al. Induction of all-female triploids in grass carp (Ctenopharyngodon idella) by integration of hormonal sex reversal and ploidy manipulation. Israel J Aquac-Bam. 2000; 52:133-150.

33. Nakamura S, Kobayashi K, Nishimura T, et al. Ovarian germline stem cells in the teleost fish, medaka (Oryzias latipes). Int J Biol Sci; in press.

34. Sánchez-Sánchez A, Camp E and Mullor J. Fishing pluripotency mechanisms in vivo. Int J Biol Sci; in press.

35. Hattori M, Hashimoto $\mathrm{H}$, Bubenshchikova E, et al. Production of fertile zebrafish nuclear transplants in non-enucleated eggs. Int J Biol Sci; in press.

36. Liu T, Hong N, Yi M, et al. Sperm nuclear transfer and transgenic production in the fish medaka. Int J Biol Sci; in press.

37. Luo $\mathrm{D}, \mathrm{Hu} \mathrm{W}$, Chen $\mathrm{S}$ et al. Critical developmental stages for the efficiency of somatic cell nuclear transfer in zebrafish. Int J Biol Sci; in press.

38. Li Z, Bhat A, Hong N, et al. Medaka cleavage embryos are capable of generating ES-like cell cultures. Int J Biol Sci; in press.

39. Zhang B, Wang $X$, Yang, et al. Establishment and characterization of a testicular cell line from the half-smooth tongue sole, Cynoglossus semilaevis. Int J Biol Sci; in press.

40. Hong N, Li Z and Hong Y. Fish stem cell cultures. Int J Biol Sci; in press.

41. Rao F, Wang T, Li Z, et al. Medaka tert expression is versatile in vivo but pluripotency-preferential in vitro. Int J Biol Sci; in press.

42. Wang D, Dwarakanath $\mathrm{M}$, Wang $\mathrm{T}$, et al. Identification of pluripotency genes in the fish medaka. Int J Biol Sci; in press.

43. Wang ZW, Ye YZ, Zhou JF, et al. Initial study of rapid establishment of inbred Hypophthalmichthys molirix and Aristichthys nobilis. Prog Nat Sci. 2003; 13(10):1104-1107.

44. Jiang Y, Liang SC, Chen BD, et al. Biological effect of heterologous sperm on gynogenetic offspring in Carassius auratus gibelio. Acta Hydro-biol Sinica. 1983; 8:1-13.

45. Zhou L, Wang Y, Gui JF. Genetic evidence for gonochoristic reproduction in gynogenetic silver crucian carp (Carassius auratus gibelio bloch) as revealed by RAPD assays. J Mol Evol. 1983; 51: 498-506.

46. Xiao YM, Liu Y, Luo C. The genetic biochemical properties of the inbreeding $F_{1}$-generation gynogenetic grass carp. J Fish, China. 2001; 25(6):495-550. 
47. Zhao J, Chen XL. Phenotypic Differentiation of offspring developed from Pengze crucian carp eggs activated by heterologous sperm. Zool Res. 2003; 24:297-301.

48. Liu SJ, Yao ZZ. Self-fertilization of Hermaphrodites of the Teleost Clarias lazerea After Oral Administration of 17-a-Methyltestosterone and Their Offspring. J Exp Zool. 1995; 273:527-532.

49. Liu SJ, Sun YD, Li SF, et al. Analysis of gonadosomatic indexes of the triploid crucian carp. J Fish China. 2002; 26(2):111-114. 